\title{
BMJ Open Outcomes of disease prevention and management interventions in food pantries and food banks: protocol for a scoping review
}

\author{
Christopher R Long, ${ }^{1}$ Brett Rowland, ${ }^{2}$ Susan C Steelman, ${ }^{3}$ Pearl A McElfish ${ }^{1}$
}

To cite: Long CR, Rowland B, Steelman SC, et al. Outcomes of disease prevention and management interventions in food pantries and food banks: protocol for a scoping review. BMJ Open 2017;7:e018022. doi:10.1136/ bmjopen-2017-018022

- Prepublication history and additional material for this paper are available online. To view please visit the journal (http:// dx.doi.org/10.1136/bmjopen2017-018022).

Received 31 May 2017

Revised 11 August 2017

Accepted 1 September 2017

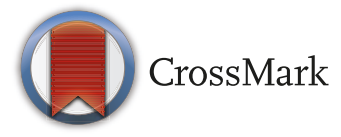

${ }^{1}$ College of Medicine, University of Arkansas for Medical

Sciences Northwest, Fayetteville, Arkansas, USA

${ }^{2}$ Office of Community Health and Research, University of Arkansas for Medical Sciences Northwest, Fayetteville, Arkansas, USA

${ }^{3}$ Division of Academic Affairs, University of Arkansas for Medical Sciences, Little Rock, Arkansas, USA

Correspondence to Dr Christopher R Long; crlong2@uams.edu

\section{ABSTRACT}

Introduction Food insecurity is a difficulty faced in many households. During periods of food insecurity, households often seek food supplied by food pantries and food banks. Food insecurity has been associated with increased risk for several health conditions. For this reason, food pantries and food banks may have great promise as intervention sites, and health researchers have begun targeting food pantries and food banks as sites for disease prevention or management interventions. The aim of the scoping review is to examine disease prevention or management interventions implemented in food pantries and food banks.

Methods and analysis Relevant electronic databases (eg, MEDLINE, Cumulative Index to Nursing and Allied Health Literature-CINAHL Complete, Science Citation Index, Cochrane Database of Systematic Reviews) will be searched for articles with a publication date of 1997 or later using Medical Subject Headings and key terms, including food aid, food banks, food pantries, food shelves, hunger, food insecurity and related concepts. For each de-duplicated study record identified by the search strategy, two reviewers will independently assess whether the study meets eligibility criteria (eg, related to intervention type, context). The reviewers will examine studies' titles, abstracts and full text, comparing eligibility decisions to address any discrepancies. For each eligible study, data extraction will be executed by two reviewers independently, comparing extracted data to address any discrepancies. Extracted data will be synthesised and reported in a narrative review assessing the coverage and gaps in existing literature related to disease prevention and management interventions implemented in food pantries.

Ethics and dissemination The review's results will be useful to healthcare practitioners who work with foodinsecure populations, healthcare researchers and food pantry or food bank personnel. The results of this scoping review will be submitted for publication to a peer-reviewed journal, and the authors will share the findings with food pantry and food bank stakeholder groups with whom they work.

\section{INTRODUCTION}

Food insecurity, defined as lack of access to sufficient amounts of nutritious food, ${ }^{1}$

\section{Strengths and limitations of this study}

- This is the first scoping review to address the literature on disease prevention and management interventions conducted in food pantries and food banks.

- The review will provide a synthesis of existing studies that will be useful to healthcare practitioners who work with food-insecure populations, healthcare researchers and food pantry and food bank personnel.

- This scoping review is focused on assessment of the coverage and gaps in the existing literature, so quality assessments of individual studies will not be a primary emphasis.

is a difficulty faced in many households. Periods of food insecurity may last for days, weeks or years for some households. During these periods, households often seek food from food pantries, which are local emergency food organisations that provide aid via distribution of unprepared food for offsite consumption. $^{2} 3$ Typical food pantries are staffed mostly by volunteers, largely funded by local donations, and associated with faithbased organisations. $^{2}$ (To minimise confusion related to regional variations in terminology, this protocol uses terminology common to the United States Department of Agriculture (USDA) reporting, where food pantries identifies local emergency food organisations that provide aid via distribution of unprepared food for offsite consumption, ${ }^{23}$ and food banks identifies organisations that exist to distribute food to food pantries and other agencies who in turn distribute directly to individuals or households.) ${ }^{2}$

In 2015, food insecurity affected approximately $12.7 \%$ of households in the USA, ${ }^{1}$ and at least $5.2 \%$ of US households obtained food from food pantries. ${ }^{3}$ Among 
food-insecure households, $28.2 \%$ obtained food from food pantries. ${ }^{3}$

Food insecurity has been associated with increased risk for several health conditions, including obesity, ${ }^{4}$ diabetes, ${ }^{56}$ hypertension ${ }^{7}$ and hyperlipidemia. ${ }^{7}$ In addition, food insecurity has been associated with inferior management of diabetes, ${ }^{6}{ }^{8}$ hypertension, ${ }^{8} \mathrm{HIV}^{8}$ and depression. ${ }^{89}$ The risk for household food insecurity rises with the presence of an adult with one or more chronic physical or mental health conditions,${ }^{10}$ and food insecurity has been identified as a predictor of adults' healthcare utilisation and costs. ${ }^{11}$ Based on these associations, health researchers have begun targeting food pantries and food banks as sites for interventions focused explicitly on preventing and managing disease. ${ }^{12-15}$

Food pantries and food banks may have great promise as intervention sites. For example, interventions targeting the kinds of food distributed from food pantries and food banks have opportunity to improve food pantry households' diet and health. Recent reviews have shown that food pantry patrons' diets often do not meet nutritional recommendations, ${ }^{16}$ and the nutritional quality of food distributed from food pantries often is insufficient to support a healthy diet. ${ }^{17}$ Furthermore, potential difficulties reaching food-insecure households at more typical sites for disease prevention and management interventions (eg, clinics, schools, churches) provide an opportunity to reach these households through food pantries and food banks. ${ }^{18}$ However, because food pantries and food banks are not typical sites for interventions explicitly targeting disease prevention and management, much remains unknown, including the range of health indicators assessed as part of these interventions, whether these interventions have been successful and what barriers and facilitators may have negatively or positively affected these interventions' potential for success. The scoping review described below will highlight what has been learnt by existing research on disease prevention and management interventions in food pantries and food banks; at the same time, it will identify gaps in this literature that can be addressed by future studies. To better contextualise the results of this review within the wider literature of disease prevention and management interventions implemented in more typical sites, the scoping review will focus on food pantry intervention studies that include assessment of change in at least one biometric health indicator (eg, body mass index, blood pressure, blood glucose).

\section{OBJECTIVES}

The aim of the scoping review is to examine the disease prevention and management interventions implemented in food pantries and food banks. The review will respond to the following questions:

1. What are the primary biometric indicators targeted by the disease prevention and management interventions implemented in food pantries and food banks, and for which biometric indicators have these interventions shown success?

2. Has the success of these interventions varied as a function of sex, ethnicity or other demographic characteristics of participants?

3. What barriers and facilitators to successful implementation of these interventions have been reported?

\section{METHODS AND ANALYSIS}

Where applicable, this protocol was designed according to the principles described in the Preferred Reporting Items for Systematic Review and Meta-Analysis Protocols statement. ${ }^{1920}$

\section{Eligibility criteria}

Studies will be selected according to the following criteria:

- Participant population: members of any racial/ethnic, sex or age group who patronise a food pantry or food bank.

- Intervention type: all types of interventions focused on disease prevention or management among food pantry or food bank patrons (eg, with respect to diabetes, obesity, infectious diseases).

- Comparator: no intervention, other intervention or within-participant preintervention.

- Outcomes: studies measuring and reporting at least one biometric indicator (eg, body mass index, blood pressure, blood glucose) as an intervention outcome variable.

- Context: interventions taking place in a food pantry or food bank or having a central component taking place in a food pantry or food bank.

- Study type: all types of studies (eg, randomised controlled trials, pilot studies, case studies).

Included studies can be from any country or region. Included studies will have been published in English with a publication date of 1997 or later. Included studies will have been published as original research in peer-reviewed journals.

Studies describing interventions focused solely on the quality of food offered in food pantries or food banks will be excluded. Likewise, as the focus of the review is on interventions in local emergency food organisations and their distributors, studies describing interventions focused solely on accessing government food aid programmes (eg, the USDA's Supplemental Nutrition Assistance Program; Women, Infants, and Children programme; or National School Lunch Program) or studies describing interventions based solely in clinical, school, workplace or home settings will be excluded.

\section{Information sources}

The following electronic databases will be searched for eligible studies published in English between 1997 and 2017: MEDLINE (OVID), MEDLINE In Process \& Daily Updates (OVID), Cumulative Index to Nursing and Allied Health Literature-CINAHL Complete (EBSCO) 
and Science Citation Index and Social Sciences Citation Index (both via Web of Science). The search of All EBM Reviews (OVID) will include Cochrane Database of Systematic Reviews, ACP Journal Club, Databases of Abstracts of Reviews of Effects, Cochrane Central Register of Controlled Trials, Cochrane Methodology Register, Health Technology Assessment and the NHS Economic Evaluation Database. All references from included studies will be reviewed for potential inclusion in the final result set. To ensure that all eligible studies are included, database searches will be updated prior to completion of the analysis.

\section{Search strategy}

The search strategy was developed by librarian coauthor SS, in consultation with coauthors CL and BR, all of whom identified key terms. Medical Subject Headings (MeSH) were used and exploded where appropriate to incorporate more specific headings under the MeSH terms. To provide a comprehensive yet focused set of results, $\mathrm{MeSH}$ terminology will be combined with advanced textword searching techniques including truncation and adjacency searching. MeSH headings chosen were Food Assistance, Food, and Food Supply. Terminology and key phrases chosen were food aid, food bags, food banks, food pantries, food shelves, soup kitchens, hunger and concepts for food insecurity. The concept of emergency food will be searched, but will not include disaster-related food services. Terminology and the overall strategy will be adapted as needed for other databases and vendor platforms. These adapted strategies and terms may be requested from the corresponding author (CL). The search terms and strategy to be used for MEDLINE (via OVID) are presented in online supplementary appendix 1 .

\section{Data management}

Search results will be exported, stored and shared among coauthors using RefWorks V.2.0, ${ }^{21}$ which is an internet-based reference management service. Removal of duplicate records from search results will be accomplished using RefWorks' duplicate-check function with manual oversight prior to duplicate removal. For the study selection process (see below), de-duplicated records will be exported from RefWorks into a Microsoft Excel 2013 V.15. $0^{22}$ spreadsheet.

\section{Study selection process}

For each de-duplicated record identified by the search strategy, two reviewers will independently examine the study's title and abstract to evaluate whether the study meets all eligibility criteria and then compare the results of the study selection process. For each study judged by the reviewers to be eligible, the full text of the article will be used to verify eligibility. Similarly, for each study for which eligibility is judged to be uncertain after examining its title and abstract, the full text of the article will be used to assess eligibility. In cases of discrepancy between the reviewers' determination of eligibility for a study, the full text of the article will be examined by both reviewers; if discrepancy remains, a third reviewer will be consulted to make a final determination.

If multiple publications of a single eligible study are identified, each of the multiple publications will be included. However, where applicable during the data extraction and synthesis process, care will be taken to avoid double-counting single studies.

\section{Data collection process}

Data extraction will be carried out by two reviewers using a Microsoft Excel spreadsheet developed for this review. Each reviewer will extract data independently from each eligible study and will then compare the results of the extraction process. Discrepancies in extracted data will be resolved through discussion and, if needed, consultation with a third reviewer.

If data extraction cannot be accomplished for an eligible study because aspects of the study are inadequately described in the full text of its article, reviewers will contact the publication's corresponding author via email (up to three attempts) in order to acquire the missing information.

If multiple publications of a single eligible study have been identified, data will be extracted from each of the multiple publications. In these cases, extracted data will be compared across publications for logical inconsistencies. If logical inconsistencies are identified, reviewers will attempt to resolve the inconsistencies through discussion or through contacting the publications' corresponding author via email (up to three attempts).

The reviewers will pilot the extraction spreadsheet on a small sample of eligible studies and will adjust the data collection fields as necessary before final data extraction begins.

\section{Data items}

The following data will be extracted from each eligible article:

- Participant population: Race/ethnicity, sex, age group, urban/rural, other participant characteristics used as an inclusion criterion.

- Intervention type: Stated disease focus of intervention (eg, prevention or management of diabetes, obesity, infectious diseases), duration of intervention and brief description of intervention.

- Comparator: Comparator type (eg, no intervention, within-participant preintervention, other intervention, other comparator) and description.

- Outcomes: Primary and secondary biometric outcomes (eg, body mass index, blood pressure, blood glucose), any other outcomes reported and the extent to which each outcome was affected by the intervention.

- Context: Whether the intervention took place in a food pantry or food bank and other notable characteristics of the food pantry or food bank (eg, did the pantry or bank follow a client-choice model of food 
distribution? Was the pantry or bank based in a religious organisation, clinic, school?).

- Barriers and facilitators of successful intervention implementation: Barriers or facilitators of intervention implementation noted by study authors, including factors related to the intervention characteristics, implementation setting, individuals involved (eg, pantry staff, research team, participant population) and implementation process. ${ }^{23}$

- Study type: Type of study design used (eg, randomised controlled trial, single-arm pilot study, single-subject design).

- Publication details: Authors, article title, journal title, year of publication, volume number, issue number, page numbers.

Because this scoping review's focus is to assess the coverage and gaps in existing literature on disease prevention and management interventions in food pantries and food banks, quality assessment of individual eligible studies will not be a primary emphasis of the data extraction process.

\section{Data synthesis}

Data synthesis will include producing quantitative summaries of extracted data that incorporate frequencies and percentages for most extracted data fields. It will also include production of qualitative summaries resulting from inductive coding of the extracted data. These summaries will be used to assess the coverage and gaps in existing literature. In addition, these summaries will allow us to identify (1) primary outcome variables of disease prevention and management interventions implemented in food pantries and food banks (and for which outcome variables these interventions have shown success); (2) the extent to which success of these interventions has varied as a function of sex, ethnicity or other demographic characteristics of participants; and (3) barriers and facilitators to successful implementation of these interventions. The data synthesis summaries will also provide enough information to determine whether the existing literature in this area would support a systematic review.

\section{Ethics and dissemination}

The purpose of this review is to examine interventions implemented in food pantries and/or food banks and intended to prevent or manage disease among people who obtain food from food pantries or food banks; it will also identify where there are gaps in the existing literature. This will be the first scoping review on this topic. For this reason, it will be useful to healthcare practitioners who work with food-insecure populations, healthcare researchers who study topics related to food insecurity or intervention implementation, food pantry or food bank personnel who explore ways to improve the health of their patrons and others. To facilitate dissemination to these groups, the results of this scoping review will be submitted for publication to a peer-reviewed journal, and the authors will share the findings with food pantry and food bank stakeholder groups with whom they work.

Contributors CL, BR and PM designed the protocol and SS developed the search strategy in consultation with $\mathrm{CL}$ and BR. CL and BR drafted the protocol and SS and PM edited the protocol. All authors approved the current version of the protocol.

Competing interests None declared.

Provenance and peer review Not commissioned; externally peer reviewed.

Open Access This is an Open Access article distributed in accordance with the Creative Commons Attribution Non Commercial (CC BY-NC 4.0) license, which permits others to distribute, remix, adapt, build upon this work non-commercially, and license their derivative works on different terms, provided the original work is properly cited and the use is non-commercial. See: http://creativecommons.org/ licenses/by-nc/4.0/

(c) Article author(s) (or their employer(s) unless otherwise stated in the text of the article) 2017. All rights reserved. No commercial use is permitted unless otherwise expressly granted.

\section{REFERENCES}

1. Coleman-Jensen A, Rabbitt MP, Gregory CA, et al. Household Food Security in the United States in 2015. US Department of Agriculture, Economic Research Service, 2016.

2. Ohls J, Saleem-Ismail F, Cohen R, et al. The Emergency Food Assistance System-findings from the provider survey, volume II: final report. Mathematica Policy Research, Inc. for the Food and Rural Economics Division, Economic Research Service, US Department of Agriculture, 2002.

3. Coleman-Jensen A, Rabbitt MP, Gregory CA, et al. Statistical supplement to Household Food Security in the United States in 2015. US Department of Agriculture, Economic Research Service 2016.

4. Pan L, Sherry B, Njai R, et al. Food insecurity is associated with obesity among US adults in 12 states. J Acad Nutr Diet 2012;112:1403-9.

5. Seligman HK, Bindman AB, Vittinghoff E, et al. Food insecurity is associated with diabetes mellitus: results from the National Health Examination and Nutrition Examination Survey (NHANES) 1999-2002. $J$ Gen Intern Med 2007;22:1018-23.

6. Laraia BA. Food insecurity and chronic disease. Adv Nutr 2013;4:203-12.

7. Seligman HK, Laraia BA, Kushel MB. Food insecurity is associated with chronic disease among low-income NHANES participants. $J$ Nutr 2010;140:304-10.

8. Wang EA, McGinnis KA, Goulet J, et al. Food insecurity and health: data from the veterans aging cohort study. Public Health Rep 2015;130:261-8.

9. Okechukwu CA, El Ayadi AM, Tamers SL, et al. Household food insufficiency, financial strain, work-family spillover, and depressive symptoms in the working class: the work, family, and health network study. Am J Public Health 2012;102:126-33.

10. Tarasuk V, Mitchell A, McLaren L, et al. Chronic physical and mental health conditions among adults may increase vulnerability to household food insecurity. J Nutr 2013;143:1785-93.

11. Tarasuk V, Cheng J, de Oliveira C, et al. Association between household food insecurity and annual health care costs. CMAJ 2015;187:E429-36.

12. Seligman HK, Lyles C, Marshall MB, et al. A Pilot Food Bank Intervention Featuring Diabetes-Appropriate Food Improved Glycemic Control Among Clients In Three States. Health Aff 2015;34:1956-63.

13. Ippolito MM, Lyles CR, Prendergast K, et al. Food insecurity and diabetes self-management among food pantry clients. Public Health Nutr 2017;20:183-9.

14. Wilson NLW, Just DR, Swigert J, et al. Food pantry selection solutions: a randomized controlled trial in client-choice food pantries to nudge clients to targeted foods. J Public Health 2017;39:fdw043.

15. Martin KS, Wu R, Wolff M, et al. A novel food pantry program: food security, self-sufficiency, and diet-quality outcomes. Am J Prev Med 2013;45:569-75.

16. Simmet A, Depa J, Tinnemann P, et al. The Dietary Quality of Food Pantry Users: A Systematic Review of Existing Literature. J Acad Nutr Diet 2017;117:563-76. 
17. Simmet A, Depa J, Tinnemann P, et al. The nutritional quality of food provided from food pantries: a systematic review of existing literature. J Acad Nutr Diet 2017;117.

18. Kaiser BL, Thomas GR, Bowers BJ. A case study of engaging hardto-reach participants in the research process: Community Advisors on Research Design and Strategies (CARDS) $($. Res Nurs Health 2017;40:70-9.

19. Moher D, Shamseer L, Clarke M, et al. Preferred Reporting Items for Systematic Review and Meta-Analysis Protocols (PRISMA-P) 2015 statement. Syst Rev 2015;4:1.
20. Shamseer L, Moher D, Clarke M, et al. Preferred reporting items for systematic review and meta-analysis protocols (PRISMA-P) 2015: elaboration and explanation. BMJ 2015;349:g7647.

21. RefWorks [computer program]. Version 2.0. Ann Arbor, Michigan, USA: ProQuest LLC, 2017.

22. Microsoft Excel [computer program]. Version 15.0. Redmond, WA: Microsoft, 2017.

23. Damschroder LJ, Lowery JC. Evaluation of a large-scale weight management program using the consolidated framework for implementation research (CFIR). Implement Sci 2013;8:51. 